\title{
Hibiscus sabdariffa L.: stabilidade da atividade antioxidante e constituintes químicos após preparo do chá
}

\author{
Hibiscus sabdariffa L.: stability of antioxidant activity and chemical \\ constituents after tea preparation
}

Recebido em: 16/02/2018 Aceito em: $\quad 02 / 05 / 2018$
Andressa Ândria Martins RIBEIRO; Diegue Henrique Nascimento MARTINS; Gabriela Roso CIBIN; Dâmaris SILVEIRA; Pérola de Oliveira MAGALHÃES; Yris Maria FONSECA-BAZZO Laboratório de Produtos Naturais, Faculdade de Ciências da Saúde, Universidade de Brasília. Campus Universitário Darcy Ribeiro. Asa Norte, CEP 70910-900.

Brasilia, Distrito Federal, Brasil.E-mail: yrisfonseca@hotmail.com

\section{ABSTRACT}

Hibiscus sabdariffa L. is used due to its peculiar taste and its healing properties. The literature reports antioxidant, anti-inflammatory and antidiabetic properties for this plant. Hibiscus is used as a tea, obtained from the infusion of parts of the plant, precisely its dried calyx. Due to its properties, the consumption of this tea in Brazil has been growing. Quality evaluation is important to guarantee the efficacy and safety of such products. In this study, three brands of H. sabdariffa L. tea were evaluated for their antioxidant properties, as well as their phytochemical profile by HPLC/ DAD. The methods of lipid peroxidation and inhibition of the formation of the phosphomolybdenum complex were used to evaluate the antioxidant activity. To better guide the safe consumption of this tea, the stability of the antioxidant activity was assessed 1 and 12 hours after preparation. In the chromatographic analysis, the presence of neochlorogenic acid was detected in all evaluated brands. The tea showed antioxidant activity in the two used methods. However, antioxidant activity tended to be lower after 12 hours of tea preparation. Therefore, to preserve the activity, the H. sabdariffa L. tea should be used up to 12 hours after preparation.

Keywords: Hibiscus sabdariffa L.; CLAE; antioxidant activity

\section{RESUMO}

A espécie vegetal Hibiscus sabdariffa L. é utilizada devido ao seu gosto peculiar e suas propriedades curativas. A literatura relata propriedades antioxidante, anti-inflamatória e antidiabética para esta planta. Sua utilização é por meio do chá, obtido da infusão de partes da planta, precisamente seu cálice seco. Devido a suas propriedades, o consumo deste chá no Brasil vem crescendo. A avaliação da qualidade é importante de forma a garantir à população a eficácia e segurança de tais produtos. Neste estudo, três marcas de chás de H. sabdariffa L. foram avaliadas quanto a suas propriedades antioxidantes, assim como seu perfil fitoquímico por CLAE/DAD. Foram utilizados os métodos de peroxidação lipídica e inibição da formação do complexo fosfomolibdênio para a avaliação da atividade antioxidante. Para melhor orientação do consumo deste chá, foi avaliada a estabilidade da atividade antioxidante após 1 e 12 horas do preparo. $\mathrm{Na}$ análise cromatográfica foi detectada a presença de ácido neoclorogênico para as três marcas avaliadas. Os chás apresentaram atividade antioxidante nos dois métodos avaliados. Contudo, a atividade antioxidante tende a reduzir após 12 horas. Esta tendência à redução de atividade possibilita a orientação para o consumo do chá de $H$. sabdariffa L. até 12 horas após preparo.

Palavras chave: Hibiscus sabdariffa L.; CLAE; atividade antioxidante. 


\section{.INTRODUÇÃO}

$\mathrm{Na}$ natureza podem ser encontrados recursos que contribuem para o aumento da perspectiva de vida da população. Embora os medicamentos convencionais estejam bem estabelecidos, a Organização Mundial de Saúde (OMS) reconhece que grande parte da população utiliza plantas e suas preparações para prevenir e tratar enfermidades, sendo ainda relatado que a popularidade da prática medicinal tradicional vem crescendo globalmente (1). Nesse cenário, o chá torna-se uma bebida popular, devido suas propriedades químicas que podem agir diretamente no corpo humano (2).

Como uma opção de chá nutritivo a partir de plantas medicinais, tem-se a espécie vegetal Hibiscus sabdariffa L. (Malvaceae), com distribuição incerta, podendo ser oriunda da Índia ou da Arábia Saudita. Suas preparações comerciais são comumente encontradas como fluído ou pó para a preparação de bebidas instantâneas ou infusões. O tratamento térmico dos seus cálices desidratados contribuem para a produção de uma bebida rica em compostos fenólicos com propriedades antioxidantes, anti-inflamatórias e antidiabéticas (3).

Hibiscus sabdariffa L. possui alto teor de vitamina A, C e E; íons de sódio e ferro (4); polissacarídeos, pectinas mucilaginosas (5) e compostos fenólicos: taninos e as antocianinas, como: delfinidina 3-xilosilglicosídeo, cianidina 3-xilosilglicosídeo, cianidina 3-glicosídeo e a delfinidina 3-glicosídeo, que são detectadas em quantidades elevadas nos cálices, sendo responsáveis por contribuir para a coloração vermelha intense do mesmo (5); além da presença de flavonoides: quercetina, luteolina, hibiscetina, sabdaretina, gossipetina. Todas substâncias citadas são consideradas como constituintes bioativos importantes no processo da ação antioxidante.

A presença de flavonoides nas amostras é de grande interesse, devido a inúmeras comprovações dos seus beneficios (6). A alimentação saudável é uma das ferramentas terapêuticas para um envelhecimento satisfatório; desse modo, muitos estudos epidemiológicos com foco em doenças crônicas concluíram que a forma mais efetiva de prevenção para uma doença específica é a prevenção primária, a qual pode ser efetuada por meio de uma nutrição balanceada (7). Essa prevenção pode ser obtida com uso de chás, como uma forma de obtenção de propriedades que podem reduzir o risco de doenças (3).

A avaliação da qualidade destes chás é importante para garantir, à população, a eficácia e segurança de tais produtos. Assim, neste estudo, três marcas de chás de H. sabdariffa L., disponíveis no mercado do Distrito Federal, Brasil, foram avaliadas quanto a suas propriedades antioxidantes, assim como seu perfil fitoquímico. É comum na prática popular preparar o chá e consumilo em pequenas tomadas ao longo do dia. Desta forma, foi avaliada também a estabilidade dos chás após 1 e 12 horas de preparo, de forma a possibilitar uma correta orientação para o consumo do chá de $H$. sabdariffa L.

\section{MATERIAL E MÉTODO}

Obtenção das amostras. Três marcas diferentes de $H$. sabdariffa L. foram adquiridas no comércio local do Distrito Federal, Brasil. As diferentes marcas foram identificadas como produto A, B e C. Todas as amostras continham o cálice seco da espécie vegetal. As amostras estavam contidas em embalagens plásticas, com descrição do modo de preparo, nome da espécie, lote, data de validade e informações sobre o distribuidor. Em apenas uma das marcas foram observadas informações nutricionais do produto.

Para a realização dos ensaios, os chás foram preparados seguindo o modo descrito na embalagem, na qual todos possuíam a mesma especificação. Foi utilizada uma colher de sobremesa ( $2 \mathrm{~g})$ do produto sobre uma xícara de chá $(150 \mathrm{~mL})$ de água fervente. O material permaneceu em infusão por 15 minutos, obtendo ao fim uma concentração de $0,013 \mathrm{~g} / \mathrm{mL}$ do chá.

Após o preparo, os chás foram transferidos e armazenados em garrafas plásticas à temperatura ambiente $\left(27^{\circ} \mathrm{C} \pm 2\right)$ até a realização dos diferentes ensaios.

\section{Cromatografia Líquida de Alta Eficiência} (CLAE). A obtenção do perfil cromatográfico por CLAE foi realizada em cromatógrafo Hitachi com fluxo de $0,6 \mathrm{~mL}$ por minuto com tempo de análise de $55 \mathrm{minu}-$ tos. Os eluentes utilizados foram ácido fosfórico $1 \% \mathrm{e}$ acetonitrila. A coluna usada no estudo foi LiChroCART 150-4,6 Purospher STAR RP-18e -5 $\mu$ m e a pré-coluna foi LiChroCART 4-4,6 Purospher STAR RP-18e $-5 \mu \mathrm{m}$. $\mathrm{O}$ intervalo de detecção foi de $230 \mathrm{a} 600 \mathrm{~nm}$, sendo as análises realizadas em 354 e $520 \mathrm{~nm}$. O software utilizado para obtenção dos dados foi o EZChrom Elite (version 3.3.2 SP1 Scientific Software, Inc.) (8).

A concentração de $5 \mathrm{mg} / \mathrm{mL}$ foi utilizada para todas as amostras que foram analisadas por CLAE, sendo as diluições obtidas com uso de água ultrapura e, em seguida, as soluções foram submetidas a filtração em membrana de $0,45 \mu \mathrm{m}$. 
Atividade antioxidante pelo método do fosfomolibdênio. $O$ ensaio foi conduzido a partir da solução reagente [fosfato de sódio tribásico 0,028 $\mathrm{M}-\mathrm{Na}_{3} \mathrm{PO}_{4} \cdot 12 \mathrm{H}_{2} \mathrm{O}$, molibdato de amônio $0,004 \mathrm{M}$ $\left(\mathrm{NH}_{4}\right)_{6} \mathrm{Mo}_{7} \mathrm{O}_{24}$ ) e ácido sulfúrico 0,6 $\left.\mathrm{M}-\mathrm{H}_{2} \mathrm{SO}_{4}\right]$ (9). Os padrões utilizados como controles positivos foram: ácido ascórbico (Fisher Scientific Company) e quercetina (Sigma), nas concentrações 0,$025 ; 0,050 ; 0,075 ; 0,100$; 0,$125 ; 0,150$ e $0,200 \mathrm{mg} / \mathrm{mL}$. Os solventes utilizados foram: água desionizada (ácido ascórbico) e etanol (quercetina).

Em tubos graduados de fundo cônico foram adicionados $100 \mu \mathrm{L}$ de cada concentração das amostras e $1000 \mu \mathrm{L}$ da solução reagente (triplicatas). $\mathrm{O}$ branco foi preparado com $100 \mu \mathrm{L}$ do solvente respectivo à amostra e $1000 \mu \mathrm{L}$ da solução reagente. Posteriormente os tubos foram dispostos em banho-maria $\left(95^{\circ} \mathrm{C}\right)$ por 90 minutos. Após resfriamento, a leitura das triplicatas foi realizada em espectrofotômetro a $695 \mathrm{~nm}$. As leituras das absorbâncias das diferentes concentrações dos padrões foram plotadas em gráfico de dispersão e a análise da regressão linear dos dados foi estabelecida. A equação da reta foi obtida, a qual foi utilizada para determinação da atividade antioxidante da amostra teste. A atividade foi expressa em equivalente de ácido ascórbico e quercetina.

Atividade antioxidante por inibição da Peroxidação Lipídica. Neste ensaio, soluções reagentes foram preparadas nas seguintes concentrações: ácido acético $20 \%$, solução de TBA $(55,50 \mathrm{mM}$ ) em SDS 1,1\% (dodecil sulfato de sódio), solução de cloreto de potássio $1,5 \%$, solução de gema de ovo $1 \%$ em cloreto de potássio, solução tampão fosfato $\left(0,02 \mathrm{~mol}\right.$ de $\mathrm{NaH}_{2} \mathrm{PO}_{4}$ e 0,01 mol de $\left.\mathrm{Na}_{2} \mathrm{HPO}_{4} \cdot 7 \mathrm{H}_{2} \mathrm{O}\right)$ com pH 7,4 e solução $A A P H$ [2,2'-azobis(2-metilpropionamidina)] (23,34 mM) em tampão fosfato. Como controle da atividade inibitória foi utilizado o BHT (hidroxitoluenobutilado) solubilizado em metanol (10). A solução mãe das amostras foram preparadas na concentração de $1 \mathrm{mg} / \mathrm{mL}$, sendo que a partir dessas soluções, a técnica de diluição seriada foi utilizada para a obtenção das concentrações: 2,$0 ; 1,8$; 1,$6 ; 1,4 ; 1,2 ; 1,0 ; 0,8$ e $0,6 \mu \mathrm{g} / \mathrm{mL}$ com a finalidade de calcular o $\mathrm{IC}_{50}$. As análises foram realizadas em triplicata $(n=3)$.

Para a reação, foram adicionados $0,1 \mathrm{~mL}$ de cada concentração de BHT ou amostras-teste, $0,5 \mathrm{~mL}$ da solução de gema de ovo, $0,4 \mathrm{~mL}$ de água destilada, $0,05 \mathrm{~mL}$ de $A A P H, 1,5 \mathrm{~mL}$ de ácido acético e $1,5 \mathrm{~mL}$ de solução TBA. Para o controle positivo foram adicionados todos os componentes menos a amostra, sendo adi- cionados no lugar da amostra $100 \mu \mathrm{L}$ de água destilada. No controle negativo não foram adicionados amostra e solução de $A A P H$, sendo adicionado, no lugar, o solvente de dissolução da amostra. Após adição dos reagentes, os tubos foram levados a banho-maria $\left(95^{\circ} \mathrm{C}\right)$ por 60 minutos. Posteriormente foram adicionados $5 \mathrm{~mL}$ de butanol e centrifugados $(11,82 \mathrm{~g})$ por 10 minutos. Em seguida, em espectrofotômetro a $533 \mathrm{~nm}$, as absorbâncias foram lidas (10).

Análise estatística. Os tratamentos dos dados obtidos nos ensaios foram realizados com a utilização do software GraphPadPrism ${ }^{\circledR}$ Version 6.0.

Para comparação entre as amostras por meio de análise de variância, após observação da distribuição dos dados (dados com distribuição não-normal), foi aplicado teste não-paramétrico, como Kruskal-Wallis - Dunn's. Os dados foram representados por mediana e intervalo inter-quartis. Foram consideradas diferenças significativas, os valores com $p<0,05$.

\section{RESULTADOS E DISCUSSÃO}

\section{Cromatografia Líquida de Alta Eficiência}

(CLAE). No perfil cromatográfico das amostras por CLAE/DAD em $354 \mathrm{~nm}$ foi observada a presença de 12 picos principais, sendo possível a identificação de ácido neoclorogênico em todas as amostras testadas (Figura 1). A identificação e a quantificação do ácido neoclorogênico foram obtidas avaliando a similaridade entre os tempos de retenção e os espectros na região de UV da amostra e o padrão de ácido neoclogênico (Sigma). Foi calculado o teor do ácido neoclorogênico nos chás a partir da equação da reta $\mathrm{y}=1,457 \mathrm{x}+0,1687(r=0,9950)$, obtida após regressão linear da área do pico do padrão. As amostras A, B e C apresentaram 3,31, 3,55 e 3,35 $\mu \mathrm{g} / \mathrm{mL}$ de ácido neoclorogênico após 1 hora de preparo (Tabela 1). Quando comparado estatisticamente (Kruskal-Wallis-Dunn's), os teores de ácido neoclorogênico após 1 hora de preparo entre as diferentes amostras testadas (A, B e C), não foi observado diferença significativa. Além disso, após 12 horas de preparo, o teor de ácido neoclorogênico se manteve para a amostra A $(100,9 \%)$ e reduziu nas amostras B e C em 12,4\% e 52,8\%, respectivamente. Apesar de ser observada redução aparente do teor de ácido neoclorogênico após 12 horas de preparo das amostras, quando realizado o teste estatístico, não foi observada diferença estatisticamente significante. Contudo, a redução apresentada pode ser importante na prática com redução da atividade biológica. 

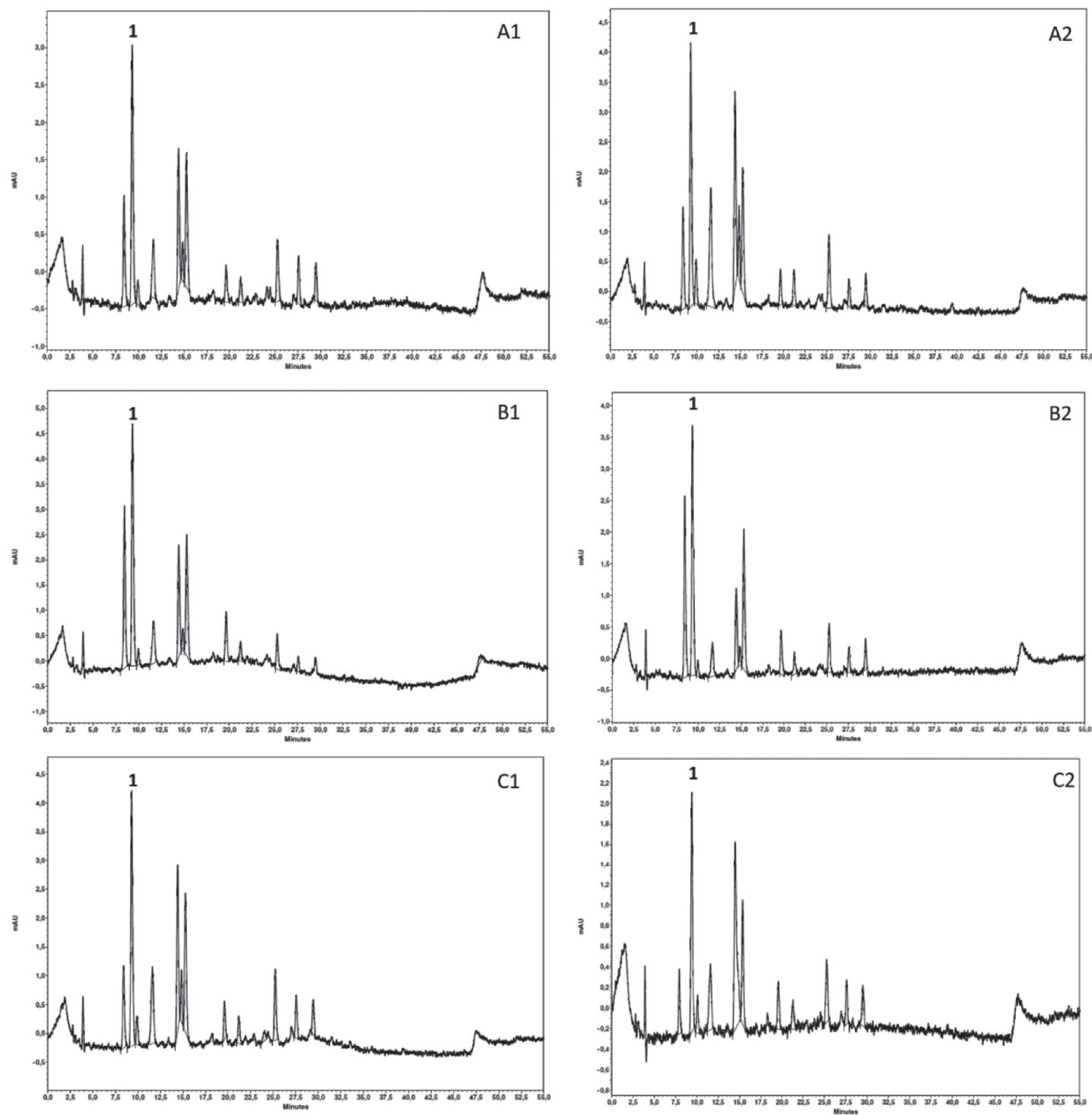

Figura 1. Perfil cromatográfico em $354 \mathrm{~nm}$ das amostras dos chás adquiridos do comércio do Distrito Federal contendo a droga vegetal de $H$. sabdariffa L. Análise realizada em Cromatógrafo Líquido de Alta Eficiência - CLAE. Condições da análise: Coluna - PurospherStar RP C18e mantida à $25^{\circ} \mathrm{C}$; Fluxo - 0,6 mL.min; Eluente - ácido fosfórico 1\% (Bomba A) e acetonitrila (Bomba B). Detector -DAD; Cromatograma em $354 \mathrm{~nm} .1$ refere-se ao ácido neoclorogênico. As letras $\mathrm{A}, \mathrm{B}$ e $\mathrm{C}$ tratam-se de marcas distintas de chás de $H$. sabdariffa. 0 número " 1 " posterior às letras $\mathrm{A}, \mathrm{B}$ e C refere-se aos chás avaliados após 1 hora de preparo e o número "2" refere-se aos chás avaliados após 12 horas.

Analisando os cromatogramas na faixa de $520 \mathrm{~nm}$, foi observado que os chás das marcas A, B e C, mantiveram qualitativamente o mesmo perfil cromatográfico quando analisadas com 1 e 12 horas após seus preparos. Os cromatogramas apresentaram 2 picos principais, sendo um deles (11,05 minutos) semelhante ao espectro encontrado na literatura como delfinidina 3-glicosídeo, composto fenólico da classe das antocianinas (Figura 2) (Tabela 2) (9). Este composto já foi previamente relatado em chás de H. sabdariffa L. segundo Fernández-Arroyo et al. (2011) (11). Atividade antioxidante pelo método do fosfomolibdênio
As diferentes amostras do chá foram analisadas quanto à atividade antioxidante pelo método de inibição da formação do complexo de fosfomolibdênio, que determina de modo quantitativo a redução de molibdênio VI para molibdênio V em pH ácido (12).

A quantificação desta atividade foi realizada por meio das curvas analíticas dos padrões de ácido ascórbico ( $\mathrm{y}=4,301 \mathrm{x}-0,02141, r=0,9980)$ e de quercetina $(\mathrm{y}=1,457 \mathrm{x}+0,1687, r=0,9950)$. Todas as amostras do chá testado apresentaram atividade antioxidante quanto à formação do complexo de fosfomolibdênio. A atividade foi expressa em equivalente de ácido ascórbico e 
quercetina. As atividades encontradas equivalentes à quercetina foram: A1 $(0,233 \mathrm{QE} / \mu \mathrm{g}), \mathrm{A} 2(0,140 \mathrm{QE} /$ $\mu \mathrm{g}), \mathrm{B} 1(0,246 \mathrm{QE} / \mu \mathrm{g}), \mathrm{B} 2(0,116 \mathrm{QE} / \mu \mathrm{g}), \mathrm{C} 1(0,138$ $\mathrm{QE} / \mu \mathrm{g})$ e $\mathrm{C} 2(0,068 \mathrm{QE} / \mu \mathrm{g})$ (Figura 3). As atividades encontradas equivalentes ao ácido ascórbico foram: A1 $(0,123 \mathrm{QE} / \mu \mathrm{g}), \mathrm{A} 2(0,091 \mathrm{QE} / \mu \mathrm{g}), \mathrm{B} 1(0,127 \mathrm{QE} / \mu \mathrm{g})$, B2 $(0,083 \mathrm{QE} / \mu \mathrm{g}), \mathrm{C} 1(0,090 \mathrm{QE} / \mu \mathrm{g})$ e $\mathrm{C} 2(0,082 \mathrm{QE} /$ $\mu \mathrm{g})$ (Figura 4).
Foram observadas reduções da atividade antioxidante pelo método do fosfomolibdênio após 12 horas do preparo. Estas reduções foram de 39,9\%, $52,9 \%$ e $50,7 \%$ para as amostras A, B e C, respectivamente, em relação ao equivalente à quercetina. Em relação ao equivalente ácido ascórbico as reduções para as amostras A, B e C foram de $26 \%, 34,6 \%$ e $11,1 \%$, respectivamente.

Tabela 1. Teor de ácido neoclorogênico por CLAE-DAD das amostras dos chás adquiridos do comércio do Distrito Federal contendo a droga vegetal de Hibiscus sabdariffa L.

\begin{tabular}{|c|c|c|c|c|}
\hline \multirow{2}{*}{$\begin{array}{c}\text { Amostras } \\
(5 \mathrm{mg} / \mathrm{mL})\end{array}$} & $\begin{array}{c}\text { Tempo de retenção } \\
(\text { Min) }\end{array}$ & \multicolumn{2}{|c|}{ Área (mAU) } & $\begin{array}{c}\text { Teor de ácido neoclorogênico } \\
(\mu \mathrm{g} / \mathrm{mL})\end{array}$ \\
\cline { 2 - 5 } & $\begin{array}{c}\text { Média } \pm \\
\text { desvio padrão }\end{array}$ & $\begin{array}{c}\text { Média } \pm \\
\text { desvio padrão }\end{array}$ & CV & $\begin{array}{c}\text { Média } \pm \\
\text { desvio padrão }\end{array}$ \\
\hline A1 & $9,31 \pm 0,02$ & $242223 \pm 41032$ & 0,1694 & $3,31 \pm 0,66$ \\
\hline B1 & $9,33 \pm 0,02$ & $256793 \pm 12161$ & 0,0474 & $3,55 \pm 0,19$ \\
\hline C1 & $9,29 \pm 0,01$ & $244479 \pm 5370$ & 0,0220 & $3,35 \pm 0,08$ \\
\hline A2 & $9,28 \pm 0,00$ & $243967 \pm 3430$ & 0,0141 & $3,34 \pm 0,05$ \\
\hline B2 & $9,33 \pm 0,01$ & $229628 \pm 6030$ & 0,0263 & $3,11 \pm 0,09$ \\
\hline C2 & $9,37 \pm 0,04$ & $135336 \pm 5607$ & 0,0414 & $1,58 \pm 0,09$ \\
\hline
\end{tabular}

Dados representam a média das triplicatas \pm DP (desvio padrão). As letras $A, B$ e $C$ representam lote/marca dos produtos adquiridos contendo a droga vegetal de $H$. sabdariffa L. 0 número " 1 " posterior às letras $A, B$ e $C$ refere-se aos chás avaliados depois de 1 hora de preparo e o número "2" refere-se aos chás avaliados após 12 horas de preparo. CV: Coeficiente de variação.

\section{Atividade antioxidante pela inibição da Perox-} idação Lipídica. O método fundamenta-se na decomposição dos corpos graxos. Deste modo, avaliou-se a atividade antioxidante de modo quantitativo por meio da reação do ácido tiobarbitúrico (TBA) com os produtos de decomposição dos hidroperóxidos. Um dos produtos dessa reação é o malonaldeído que reage com o TBA formando um complexo de cor vermelha. A reação acontece em meio ácido e em altas temperaturas para aumentar a velocidade e sensibilidade do teste (13).

Todas as amostras testadas apresentaram atividade de inibição da peroxidação lipídica. As atividades encontradas foram: A1 $\left(\mathrm{IC}_{50}: 1,84 \mu \mathrm{g} / \mathrm{mL}\right), \mathrm{A} 2\left(\mathrm{IC}_{50}: 1,83\right.$ $\mu \mathrm{g} / \mathrm{mL}), \mathrm{B} 1\left(\mathrm{IC}_{50}: 1,31 \mu \mathrm{g} / \mathrm{mL}\right), \mathrm{B} 2\left(\mathrm{IC}_{50}: 1,68 \mu \mathrm{g} / \mathrm{mL}\right)$, $\mathrm{C} 1\left(\mathrm{IC}_{50}: 2,68 \mu \mathrm{g} / \mathrm{mL}\right)$ e $\mathrm{C} 2\left(\mathrm{IC}_{50}: 2,81 \mu \mathrm{g} / \mathrm{mL}\right)$; enquanto o $\mathrm{IC}_{50}$ do padrão BHT foi de $1,87 \mu \mathrm{g} / \mathrm{mL}$ (Figura 5). Não foram observadas diferenças estatisticamente significativas entre as amostras analisadas após o preparo de 1 e 12 horas.

A possível presença de constituintes como ácido neoclorogênico, antocianidinas, entre outros flavo- noides podem explicar as propriedades terapêuticas do chá de $H$. sabdariffa L. Corroborando com este preposto, a literatura relata a atividade antioxidante e anti-inflamatória para o ácido neoclorogênico. Em relação à atividade antioxidante, o ácido neoclorogênico atua como receptor de radicais livres e na atividade anti-inflamatória, ele inibe citocinas (14). As antocianinas são responsáveis pela coloração vermelha dos cálices de $H$. sabdariffa L. e também conferem atividade antioxidante, o que possibilita o bloqueio de radicais livres $(9,15,16)$.

Os resultados obtidos da análise por CLAE/DAD e da atividade antioxidante pelo método fosfomolibdênio indicam instabilidade do chá, pois foram observadas reduções da atividade antioxidante e teor de ativos após 12 horas do preparo. Mesmo não sendo observada significância estatística dos dados, os resultados mostram uma tendência que não pode ser descartada. Sugere-se que o usuário consuma o chá no momento do preparo, pois 12 horas após a preparação suas propriedades químicas tendem a sofrer alterações. 


\section{CONCLUSÃO}

A análise dos chás contendo os cálices de $H$. sabdariffa L. mostrou a presença de componentes com propriedades antioxidantes, tais como ácido neoclorogênico e antocianidinas. $\mathrm{O}$ estudo sugere que após 12 horas do preparo os chás podem passar por alterações químicas, resultando em redução da atividade antioxidante, o que pode culminar em redução das propriedades medicinais. Esta tendência à redução de atividade observada possibilita a orientação para o consumo do chá de H. sabdariffa L. em até 1 hora após o preparo.
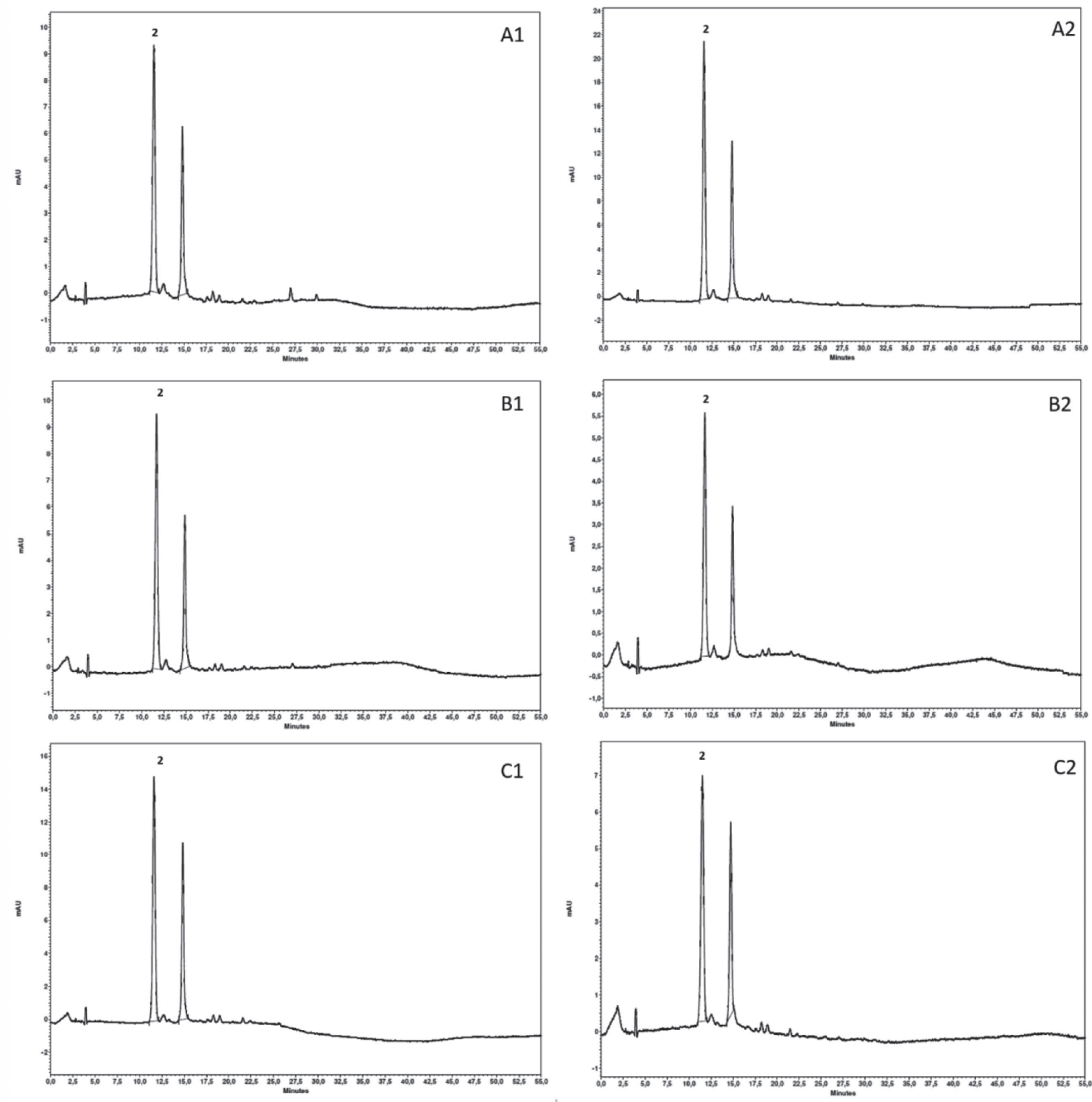

Figura 2. Perfil cromatográfico em $520 \mathrm{~nm}$ das amostras dos chás das amostras adquiridas do comércio do Distrito Federal contendo a droga vegetal de H. sabdariffa L. Análise realizada em Cromatógrafo Líquido de Alta Eficiência - CLAE. Condições da análise: Coluna - PurospherStar RP $C_{18}$ e mantida à $25^{\circ} \mathrm{C}$; Fluxo - 0,6 mL.min; Eluente - ácido fosfórico 1\% (Bomba A) e acetonitrila (Bomba B). Detector -DAD; Cromatograma em 520 nm. 2 - refere-se ao possível pico da delfinidina 3-glicosídeo, conforme espectro semelhante descrito por SKAAR e cols. (2014) (17). As letras A, B e C tratam-se de marcas distintas de chás de $H$. sabdariffa. 0 número "1" posterior às letras A, B e C refere-se aos chás avaliados após 1 hora de preparo e o número "2" refere-se aos chás avaliados após 12 horas. 
Tabela 2. Dados obtidos na análise dos chás das amostras adquiridas contendo a droga vegetal Hibscus sabdariffa L. em $520 \mathrm{~nm}$ por CLAE-DAD.

\begin{tabular}{|c|c|c|c|}
\hline \multirow{2}{*}{$\begin{array}{c}\text { Amostras } \\
(\mathbf{5} \mathrm{mg} / \mathrm{mL})\end{array}$} & $\begin{array}{c}\text { Tempo de retenção }(\mathrm{min}) \\
\text { Área (mAU) }\end{array}$ \\
\hline A1 & $\begin{array}{c}\text { Média } \pm \\
\text { desvio padrão }\end{array}$ & $\begin{array}{c}\text { Média } \pm \\
\text { desvio padrão }\end{array}$ \\
\hline B1 & $11,72 \pm 0,09$ & $737888 \pm 17406$ & 0,0236 \\
\hline C1 & $11,64 \pm 0,02$ & $741513 \pm 1904$ & 0,0026 \\
\hline A2 & $11,57 \pm 0,05$ & $1156022 \pm 2081$ & 0,0018 \\
\hline B2 & $11,58 \pm 0,01$ & $1697067 \pm 15923$ & 0,0094 \\
\hline C2 & $11,65 \pm 0,00$ & $436784 \pm 3712^{*}$ & 0,0085 \\
\hline
\end{tabular}

Dados representam a média das triplicatas \pm desvio padrão. As letras A, B e C representam lote/marca dos produtos adquiridos contendo a droga vegetal de H. sabdariffa L. 0 número " 1 " posterior às letras $A, B$ e $C$ refere-se aos chás avaliados após 1 hora de preparo e o número " 2 " refere-se aos chás avaliados após 12 horas de preparo. CV: Coeficiente de variação. * $p<0,05$ em comparação a A2 por Kruskal-Wallis - Dunn's.

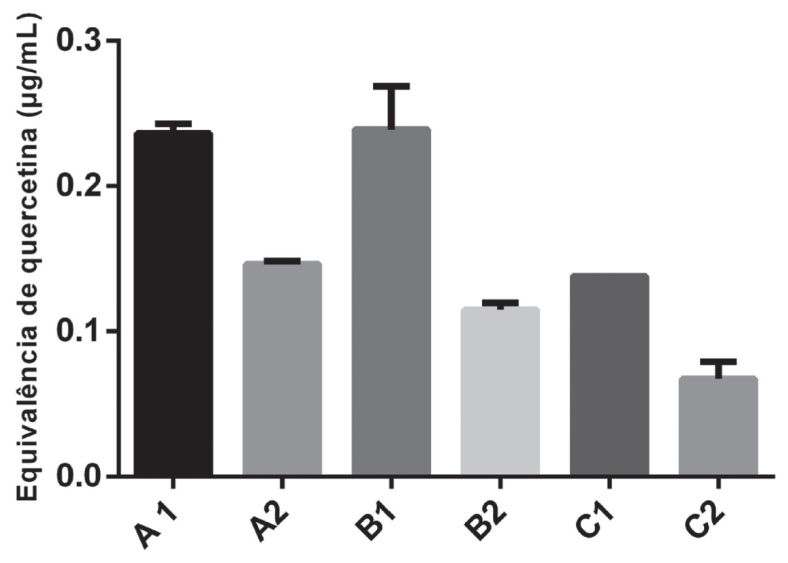

Figura 3. Atividade antioxidante expressa em equivalência de quercetina por inibição da formação do complexo fosfomolibdênio para os chás de Hibiscus sabdariffa L. Os dados foram representados por mediana das triplicatas \pm intervalo interquartis, sendo considerados diferentes os dados que apresentaram $p<0,05$. Aplicou-se teste Kruskal-Wallis - Dunn's para a comparação da atividade antioxidante (equivalente de quercetina) para os chás (marcas distintas) com análise após 1 e 12 horas de preparo. As letras A, B e C tratam-se de marcas distintas de chás de $H$. sabdariffa $\mathrm{L}$. 0 número " 1 " posterior as letras às $A, B$ e $C$ refere-se aos chás avaliados após 1 hora de preparo e o número "2" refere-se aos chás avaliados após 12 horas.

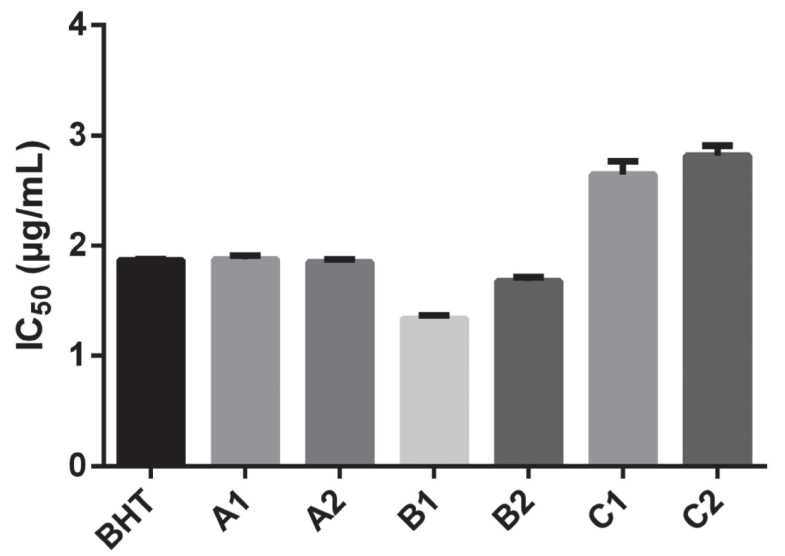

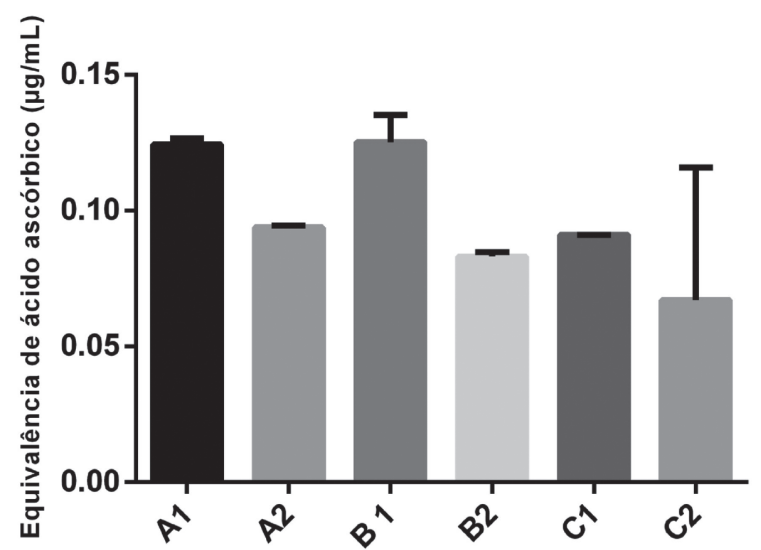

Figura 4. Atividade antioxidante expressa em equivalência de ácido ascórbico por inibição da formação do complexo fosfomolibdênio para os chás de Hibiscus sabdariffa L. Os dados foram representados por mediana das triplicatas \pm intervalo interquartis, sendo considerados diferentes os dados que apresentaram $p<0,05$. Aplicou-se teste Kruskal-Wallis - Dunn's para a comparação da atividade antioxidante (equivalente de ácido ascórbico) para os chás (marcas distintas) com análise após 1 e 12 horas de preparo. As letras A, B e C tratam-se de marcas distintas de chás de $H$. sabdariffa L. 0 número " 1 " posterior às letras $A, B$ e $C$ refere-se aos chás avaliados após 1 hora de preparo e 0 número " 2 " refere-se aos chás avaliados após 12 horas.

Figura 5. Atividade antioxidante por inibição da peroxidação lipídica para os chás de Hibiscus sabdariffa L. Os dados foram representados por mediana das triplicatas \pm intervalo interquartis, sendo considerados diferentes os dados que apresentaram $p<0,05$. Aplicou-se teste Kruskal-Wallis- Dunn's para a comparação do IC $_{50}$ obtidos para os chás com análise após 1 e 12 horas de preparo e BHT. As letras A, B e C tratam-se de marcas distintas de chás de $H$. sabdariffa L. 0 número " 1 " posterior às letras $A, B$ e $C$ refere-se aos chás avaliados após 1 hora de preparo e o número "2" refere-se aos chás avaliados após 12 horas. 
$0.14450 / 2318-9312 . v 30 . e 2 . a 2018 . p p 102-109$

\section{REFERÊNCIAS}

1. BRASIL. Política e Programa Nacional de Plantas Medicinais e Fitoterápicos. Brasília, Ministério da Saúde; 2016.

2. Marín SLA, Pereira D, Carminatti R, Oldoni TLC, Rodrigues MB. Extração, identificação e quantificação de cafeína em chá composto (Camellia sinensis e Hibiscus sabdariffa L.) por cromatografia líquida de alta eficiência (CLAE). Syn scy UTFPR. 2015;10(1):59-66.

3. Rocha IC, Bonnlaender B, Sieversc H, Pischelac I, Heinricha M. Hibiscus sabdariffa L. - A phytochemical and pharmacological review. Food Chem. 2014;165:424443. DOI: 10.1016/j.foodchem.2014.05.002

4. Iyare EE, Adegoke OA, Nwagha UI. Mechanism of the decreased food consumption and weight gain in rats following consumption of aqueous extract of the calyx of Hibiscus sabdariffa during pregnancy. Asian Pacific journal of tropical medicine. 2010;3(3):185-188. DOI: 10.1016/S1995-7645(10)60005-3

5. Borrás-Linares I, Herranz-López M, Barrajón-Catalán E, Arráez-Román D, Gonzálezlvarez I, Bermejo M, et al. Permeability Study of Polyphenols Derived from a Phenolic-Enriched Hibiscus sabdariffa Extract by UHPLC-ESI-UHR-Qq-TOF-MS. Int J Mol Sci. 2015;16(8): 18396-18411. DOI: 10.3390/ijms160818396

6. Latorre A, Bloise MC, Colosimo C, Biasio FD, Defazio $\mathrm{G}$, Berardelli A, et al. Dyskinesias and motor symptoms onset in Parkinson disease. Parkinsonism and Related Disorders. 2014;20(12):1427-1429. DOI: $10.1016 /$ j. parkreldis.2014.10.002

7. Sichieri R, Coitinho DC, Monteiro JB, Coutinho WF. Recomendações de alimentação e nutrição saudável para a população brasileira. Arq Bras Endocrinol Metab. 2000;44(3):227-232. DOI: 10.1590/S000427302000000300007

8. Leite CFM, Leite BHM, Barros IMC, Gomes SM, Fagg CW, Simeoni LA, et al. Determination of rutin in Erythroxylum suberosumextract by liquid chromatography: applicability in standardization of herbs and stability studies. Bol latinoam Caribe plantas med aromát. 2014;13(2):134-143.

9. Freitag N, Zwier MV, Barrientos G, Tirado-González I, Conrad ML, Rose M, et al. Influence of relative NK-DC abundance on placentation and its relation to epigenetic programming in the offspring. Cell Death and Disease. 2014;5(8):1-8. DOI: 10.1038/cddis.2014.353

10. Morais SM, Catunda FEA, Silva ARA, Neto JSM, Rondina D, Cardoso JHL. Atividade antioxidante de óleos essenciais de espécies de Croton do Nordeste do Brasil. Quim Nova. 2006;29(5):907-10. DOI: 10.1590/S010040422006000500004.

11. Fernández-Arroyo S, C.Rodríguez-Medina I, Beltrán-Debón R, Pasini F, Joven J, Micol V, et al. Quantification of the polyphenolic fraction and in vitro antioxidant and in vivo anti-hyperlipemic activities of Hibiscus sabdariffa aqueous extract. Food Res International. 2011;44(5):1490-1495. DOI: $10.1016 / j$. foodres.2011.03.040

12. Prieto P, Pineda M, Aguilar M. Spectrophotometric Quantitation of Antioxidant Capacity through the formation of a phosphomolybdenum complex: specific application to the determination of vitamin E. Anal Biochem. 1999;269(2):337-341. DOI: 10.1006/abio.1999.4019

13. Borges LL, Lúcio TC, Gil ES, Barbosa EF. Uma abordagem sobre métodos analíticos para determinação da atividade antioxidante em produtos naturais. En Biosfera. 2011;7(12):1-20.

14. Maria CAB, Moreira RFA. Métodos para análise de ácido clorogênico. Quim Nova. 2004;27(4):586-592. DOI: 10.1590/S0100-40422004000400013.

15. Malacrida CR, Motta S. Antocianinas em suco de uva: composição e estabilidade. B CEPPA. 2006;24(1):59-82. DOI: 10.5380/cep.v24i1.5294

16. Ramos DD, Vieira MC, Formagio ASN, Cardoso CAL, Ramos DD, Carnevali TO. Atividade antioxidante de Hibiscus sabdariffa L. em função do espaçamento entre plantas e da adubação orgânica. C Rural. 2011;41(8):13311336. DOI: $10.1590 / \mathrm{S} 0103-84782011005000107$.

17. Skaar I, Adaku C, Jordheim M, Byamukama R, Kiremire $\mathrm{B}$, Andersen ØM. Purple anthocyanin colouration on lower (abaxial) leaf surface of Hemigraphis colorata (Acanthaceae). Phytochemistry. 2014;105:141-146. DOI: $10.1016 /$ j.phytochem.2014.05.016 